\title{
Exhaled nitric oxide during infancy as a risk factor for asthma and airway hyperreactivity
}

\author{
Daniel Chang ${ }^{1}$, Weiguo Yao ${ }^{1}$, Christina J. Tiller ${ }^{1}$, Jeffrey Kisling ${ }^{1}$, \\ James E. Slaven ${ }^{2}$, Zhangsheng Yu², Mark H. Kaplan ${ }^{1,3}$ and Robert S. Tepper ${ }^{1}$
}

Affiliations:

${ }^{1}$ Dept of Pediatrics, James Whitcomb Riley Hospital for Children, Herman B. Wells Center for Pediatric Research, Indiana University School of Medicine, Indianapolis, IN, USA.

${ }^{2}$ Dept of Biostatistics, Indiana University School of Medicine, Indianapolis, IN, USA.

${ }^{3}$ Dept of Microbiology and Immunology, Indiana University School of Medicine, Indianapolis, IN, USA.

\section{Correspondence:}

Robert S. Tepper, Dept of Pediatrics, Indiana University Medical Center, James Whitcomb Riley Hospital for Children, 702 Barnhill Drive, ROC 4270, Indianapolis, IN 46202-5225, USA.

E-mail: rtepperdiupui.edu

ABSTRACT Childhood asthma is often characterised by elevated exhaled nitric oxide (eNO), decreased lung function, increased airway reactivity and atopy; however, our understanding of when these phenotypic airway characteristics develop remains unclear. This study evaluated whether eNO, lung function, airway reactivity and immune characteristics during infancy are risk factors of asthma at age 5 years.

Infants with eczema, enrolled prior to wheezy illness $(n=116)$, had eNO, spirometry, airway reactivity and allergen sensitisation assessed at entry to the study and repeated at age 5 years $(n=90)$.

Increasing eNO at entry was associated with an increased risk of asthma $(\mathrm{p}=0.037)$ and increasing airway reactivity $(\mathrm{p}=0.015)$ at age 5 years. Children with asthma at 5 years of age had a greater increase in eNO between infancy and age 5 years compared with those without asthma $(p=0.002)$. Egg sensitisation at entry was also associated with an increased risk of asthma $(p=0.020)$, increasing eNO $(p=0.002)$ and lower forced expiratory flows ( $\mathrm{p}=0.029$ ) as a 5 year-old.

Our findings suggest that, among infants at high risk for developing asthma, eNO early in life may provide important insights into the subsequent risk of asthma and its airway characteristics.

@ERSpublications

Increased eNO in infants with eczema was associated with increased asthma risk and airway reactivity at age 5 years http://ow.ly/AJcEd

For editorial comments see Eur Respir J 2015; 45; 30-32 [DOI: 10.1183/09031936.00201814].

Received: Feb 202014 | Accepted after revision: Aug 182014 | First published online: Sept 262014

Support statement: Supported by NIH grant numbers HL080071, AI070448 and AI057459.

Conflict of interest: None declared.

Copyright OERS 2015 


\section{Introduction}

Childhood asthma is often characterised by decreased lung function, heightened airway reactivity to bronchoconstrictors and elevated exhaled nitric oxide (eNO) [1-3]. However, the relationship between these airway characteristics of asthma early in life and the development of childhood asthma remains unclear. Airway inflammation and lung function measurements in infants can improve our understanding of the onset and progression of airway disease and may be useful in assessing the risk for developing asthma. While there have been relatively few longitudinal studies assessing airway physiology in infants prior to the onset of episodes of wheezing, the use of forced expiratory flows (FEF) has enabled a similar methodology to be applied longitudinally from infancy into adulthood and has provided the most important insights into the development of airway disease associated with childhood asthma [4-8].

eNO has been used as a biomarker for eosinophilic airway inflammation, but there has not been a longitudinal study of eNO from infancy, prior to onset of respiratory symptoms, into childhood. While several tidal breathing techniques have assessed eNO in infants $[9,10]$, our laboratory developed a technique to measure eNO in sedated infants under conditions of constant flow from elevated lung volume, a methodology similar to that used in older co-operative children and adults [11-13]. We previously reported that among a cohort of infants enrolled with eczema prior to the onset of wheezing increased eNO levels were associated with increased airway reactivity [14]. Those infants sensitised to egg and/or milk also had lower FEF and heightened airway reactivity. Our cohort has been reassessed at 5 years of age and we hypothesised that higher eNO, lower FEF and greater airway reactivity upon entry to the study would be associated with an increased risk of asthma at 5 years of age. We also hypothesised that those with asthma at age 5 years would have a greater increase in eNO, a smaller increase in FEF, and a greater increase in airway reactivity between infancy and follow-up when compared with subjects without asthma.

\section{Methods}

Subjects

Full-term infants and toddlers with eczema $(n=116)$ were recruited from general paediatric clinics and advertisements, and were excluded if a history of wheezing or treatment with asthma medications was present [15]. Subjects were evaluated at entry to the study (median age of 10.7 months, range 2.619.1 months) and at 5 years of age $(n=90)$. The study was approved by the Institutional Review Board (Indiana University School of Medicine, Indianapolis, IN, USA) and written parental consent was obtained.

\section{Airway function}

Exhaled nitric oxide

In infants, eNO was measured online at a constant expiratory flow from raised lung volume [11]. In 5 -year-olds, eNO was measured online with a Niox eNO analyzer (Aerocrine, Solna, Sweden) [12, 13].

\section{Spirometry}

In infants, FEF were obtained using the raised volume technique [15]. Forced vital capacity (FVC) and FEF at $25-75 \%$ of FVC (FEF25-75\%) were expressed as z-scores using normative data from our laboratory [15]. In 5-year-olds, spirometry was performed by subjects; FVC and FEF25-75\% were expressed as z-scores using normative data from our laboratory [16].

\section{Airway resistance and reactance}

In 5-year-olds, airway resistance and reactance at $5 \mathrm{~Hz}$ (R5; X5) were obtained by impulse oscillometry (IOS) according to American Thoracic Society (ATS) and European Respiratory Society guidelines [17].

\section{Airway reactivity}

In infants, airway reactivity to increasing concentrations of inhaled methacholine ( $\mathrm{MCh}$ ) was assessed using FEF and quantified by PC30 (the provocative concentration decreasing baseline FEF75\% by 30\%) [14].

In 5-year-olds, airway reactivity was assessed using IOS with increasing inhaled $\mathrm{MCh}\left(0.0625 \mathrm{mg} \cdot \mathrm{mL}^{-1}\right.$; $0.25 \mathrm{mg} \cdot \mathrm{mL}^{-1} ; 1.0 \mathrm{mg} \cdot \mathrm{mL}^{-1} ; 4.0 \mathrm{mg} \cdot \mathrm{mL}^{-1}$; and $16 \mathrm{mg} \cdot \mathrm{mL}^{-1}$ ) using a five-breath technique according the ATS guidelines [18]. Airway reactivity was quantified by PC40,R5 (the MCh dose that increased baseline R5 by $40 \%)$ or the final MCh concentration inhaled [19, 20]. Following the last MCh dose and R5 measurement, spirometry was repeated and the percentage decrease in forced expiratory volume in $1 \mathrm{~s}$ $(\mathrm{FEV} 1)$ from baseline $(\% \Delta \mathrm{FEV} 1)$ was used as an index of airway reactivity.

\section{Immune characteristics}

$\lg E$

Venous blood was obtained at study entry and at 5 years of age to assess total IgE and allergen-specific IgE (egg white, milk, wheat, cat, house dust mite, Timothy grass, Bermuda grass, ragweed, Alternaria 
species and Mountain cedar; ImmuneTech Inc., Foster City, CA, USA). Subjects were consider allergen sensitised when the specific IgE level was $>0.35 \mathrm{IU} \cdot \mathrm{mL}^{-1}$.

Cytokines

Peripheral blood mononuclear cells (PBMC) were isolated from the venous blood, cultured and stimulated with PMA (12-O-tetradecanoylphorbol 13-acetate)/ionomycin to assess cytokine production (interleukin (IL)-4, IL-5, IL-13, IL-17, IL-9, IL-10 and interferon (IFN) $\gamma$ ) [21]. The ratios of individual cytokines to IFN $\gamma$ were employed to assess the balance between the T-helper cell (Th)2 and Th1 responses [21, 22].

\section{Eczema}

Eczema was doctor-diagnosed and the severity quantified using Scoring Atopic Dermatitis (SCORAD) at study entry and at age 5 years [23].

\section{Respiratory history and asthma diagnosis}

Histories for maternal cigarette smoking during pregnancy, cigarette smoking by household members or caregivers, as well as family (parent/sibling) history of asthma and/or allergy were obtained at study visits. In 5-year-olds, current asthma was defined as: 1) physician diagnosis of asthma at any time and a history of wheezing in the previous 12 months, or 2) the use of asthma medication (bronchodilators or inhaled corticosteroids) in the previous 12 months.

\section{Statistical analysis}

Patient characteristics (demographics, sensitisation variable, cytokines, eNO and lung functions) at entry were summarised for those with and without follow-up at 5 years of age, and compared using two-sample t-tests for continuous variables and Chi-squared test or Fisher's exact test for categorical variables. Total serum IgE and cytokine measurements were natural log transformed and satisfied the normal distribution assumption using Kolmogorov-Smirnov test.

Means of eNO, z-FEF25-75\% and airway reactivity were plotted at study entry and 5 years of age by asthma status at 5 -years-old. t-tests were used to test the difference at each time point and the changes over time between the two groups. Using a logistic regression model we evaluated the individual associations between eNO, z-FEF25-75\% and airway reactivity at entry as predictors and the response variable of asthma status at 5 years of age, adjusting for age at entry to the study, race, sex and smoking during pregnancy. Results of each logistic regression model were reported as odds ratio with $95 \%$ confidence intervals and p-values.

Linear regression models were used to evaluate the association between each predictor of interest at entry (sensitisation to each individual allergen, total IgE, cytokines and SCORAD) and each response variable at 5 years of age (eNO, z-FEF25-75\% and $\% \Delta \mathrm{FEV} 1)$ with adjustments for age at entry to the study, race, sex and smoking during pregnancy. Results of each linear regression model were presented as a coefficient estimate with $95 \%$ confidence interval and p-value. For the cytokine production, we standardised using standard deviation as $\log ($ cytokine +1$) / \mathrm{SD} \times($ cytokine +1$)$; the estimated coefficient represents the change in mean response or log odds ratio corresponding to one standard deviation increase in the predictor. All analyses were performed using SAS v9.3 (SAS Institute, Cary, NC, USA).

\section{Results}

Subjects

Of 116 subjects recruited at entry, 26 subjects were lost to follow-up at 5 years of age (table 1). Those who did not have follow-up were predominantly female ( $70 \%$ versus $47 \% ; \mathrm{p}=0.035)$, non-Caucasian ( $76 \%$ versus $48 \% ; \mathrm{p}=0.017$ ), more likely to have history of maternal smoking during pregnancy (25\% versus $7 \%$; $\mathrm{p}=0.013)$ and less likely to have family asthma (69\% versus $86.5 \%$; $\mathrm{p}=0.048)$. Among 5 -year-olds, $61(68 \%)$ out of 90 subjects had asthma.

\section{Exhaled nitric oxide}

Subjects with asthma at 5 years of age had significantly higher eNO at study entry as infants prior to any wheezing (eNO difference: $3.5 \mathrm{ppb}, 95 \%$ CI $0.12-6.84 \mathrm{ppb}$; $\mathrm{p}=0.035$ ), as well as a significantly higher eNO as 5-year-olds compared with subjects without asthma (eNO difference: $10.8 \mathrm{ppb}, 95 \%$ CI 1.53-19.99 ppb; $\mathrm{p}=0.023$ ). In addition, the increase in eNO between infancy and follow-up at 5 years of age was significantly greater for subjects with asthma as 5-year-olds compared with subjects without asthma (slope difference: 7.3 , 95\% CI 6.9-7.7; p=0.001) (fig. 1). Higher eNO at study entry was significantly associated with a greater risk of asthma at 5 years of age; each ppb increase in eNO at entry was associated with an increased risk of asthma at 5 years of age (OR 1.13, 95\% CI 1.01-1.26; p=0.037). Increasing eNO at study entry was also significantly associated with greater airway reactivity at 5 years of age; each ppb increase in 


\section{TABLE 1 Baseline characteristics}

\begin{tabular}{|c|c|c|c|c|}
\hline & All & LTFU & Followed up & p-value ${ }^{\#}$ \\
\hline Subjects $\mathrm{n}$ & 116 & 26 & 90 & \\
\hline Age months & $10.66 \pm 4.62$ & $9.90 \pm 4.64$ & $10.92 \pm 4.61$ & 0.301 \\
\hline Sex (female) & $63(52.5)$ & $21(70.0)$ & $42(46.7)$ & 0.035 \\
\hline Race (white) & 53 (45.3) & $7(24.1)$ & 46 (52.3) & 0.009 \\
\hline Smoking during pregnancy & $13(11.0)$ & 7 (25.0) & $6(6.7)$ & 0.013 \\
\hline Family history of asthma & $97(82.2)$ & $20(69.0)$ & $77(86.5)$ & 0.048 \\
\hline eNO ppb & $13.1 \pm 6.82$ & $10.9 \pm 5.60$ & $13.8 \pm 7.03$ & 0.060 \\
\hline z-FEF25-75\% & $-0.85 \pm 1.10$ & $-1.15 \pm 1.28$ & $-0.77 \pm 1.03$ & 0.126 \\
\hline Egg allergen sensitisation & $19(16.7)$ & $2(7.7)$ & $17(19.3)$ & 0.234 \\
\hline Aeroallergen sensitisation" & $31(27.2)$ & $7(26.9)$ & $24(27.3)$ & 0.999 \\
\hline Total $\lg \mathrm{E}^{+}$ & $2.20 \pm 1.67$ & $1.94 \pm 1.33$ & $2.27 \pm 1.76$ & 0.375 \\
\hline IL-4/IFN $\gamma^{\S}$ & $0.74 \pm 0.72$ & $0.70 \pm 0.90$ & $0.76 \pm 0.65$ & 0.765 \\
\hline IL-5/IFN $\gamma^{\S}$ & $0.85 \pm 0.81$ & $0.84 \pm 0.78$ & $0.90 \pm 0.90$ & 0.744 \\
\hline IL-9/IFN $\gamma^{\S}$ & $0.72 \pm 0.82$ & $0.86 \pm 0.90$ & $0.68 \pm 0.79$ & 0.336 \\
\hline IL-10/IFN $\gamma^{\S}$ & $0.54 \pm 0.55$ & $0.60 \pm 0.79$ & $0.52 \pm 0.45$ & 0.598 \\
\hline IL-13/IFN $\gamma^{\S}$ & $1.02 \pm 0.89$ & $1.17 \pm 0.91$ & $0.97 \pm 0.89$ & 0.325 \\
\hline IL-17/IFN $\gamma^{\S}$ & $1.13 \pm 0.82$ & $1.36 \pm 0.86$ & $1.05 \pm 0.80$ & 0.098 \\
\hline SCORAD & $10.28 \pm 7.70$ & $9.59 \pm 8.51$ & $10.49 \pm 7.47$ & 0.598 \\
\hline
\end{tabular}

Data are presented as mean \pm SD or $\mathrm{n}(\%)$, unless otherwise stated. Bold font indicates statistical significance. LTFU: lost to follow-up; eNO: exhaled nitric oxide; z-FEF25-75\%: z-score for forced expiratory flows at $25-75 \%$ of forced vital capacity; IL: interleukin; IFN: interferon; SCORAD: scoring atopic dermatitis. " : p-values are for comparison between LTFU and patients who were followed at 5 years of age, two-sample T-tests were used for continuous variables and Fisher's exact test for categorical variables; I: presence of sensitisation to at least one of the following allergens: Timothy grass, Bermuda grass, short ragweed, Alternaria species and/or Mountain cedar; ${ }^{+}$: total serum IgE expressed as the log transformed value; ${ }^{\S}$ : standardised as log(cytokine +1$) /$ sD $\times($ cytokine +1$)$.

eNO at study entry was associated with a greater decrease in FEV1 during the $\mathrm{MCh}$ challenge as 5-year-olds (coefficient $0.76 \%, 95 \%$ CI $0.71-0.85 \%$; $\mathrm{p}=0.015$ ).

\section{Forced expiratory flows}

Subjects with asthma at 5 years of age had a statistically nonsignificant lower z-FEF25-75\% at entry to the study ( $\mathrm{z}$-score difference: $0.46,95 \% \mathrm{CI}-0.01-0.93 ; \mathrm{p}=0.053$ ) and had significantly lower $\mathrm{z}$-FEF $25-75 \%$ as

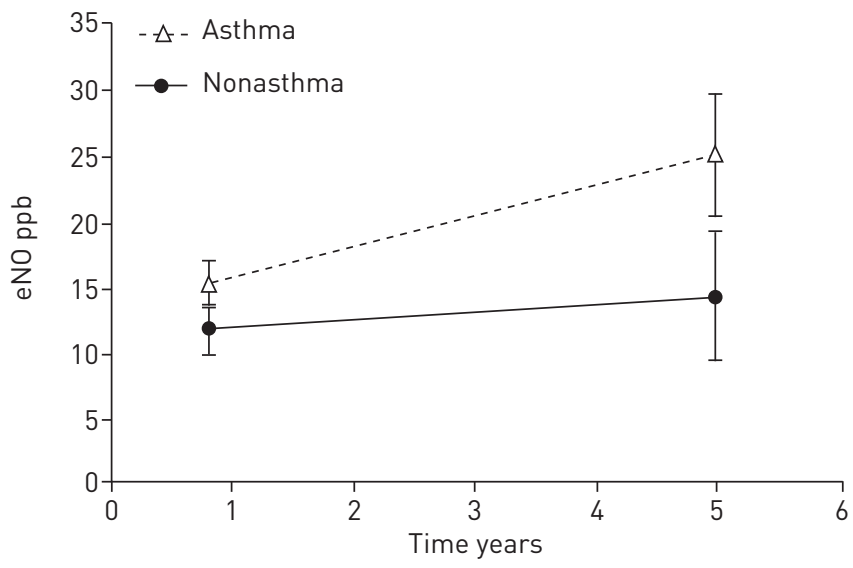

FIGURE 1 Exhaled nitric oxide (eNO) at study entry and at 5 years of age. Subjects with asthma at 5 years of age had significantly higher eNO at entry to the study as infants prior to any wheezing (eNO difference: $3.5 \mathrm{ppb}, 95 \% \mathrm{CI} 0.12-$ $6.84 \mathrm{ppb} ; \mathrm{p}=0.035$ ), as well as a significantly higher eNO at 5 years of age compared with subjects without asthma (eNO difference: $10.8 \mathrm{ppb}, 95 \%$ CI 1.53-19.99 ppb; $\mathrm{p}=0.023$ ). In addition, the increase in eNO between infancy and 5-year-old follow-up was significantly greater for subjects with asthma at 5 years of age compared with subjects without asthma (slope difference: 7.3, 95\% CI 6.9-7.7; $\mathrm{p}=0.001$ ). Data are presented as mean \pm SE. The model was adjusted by age at entry to the study, sex, race and smoking during pregnancy. 


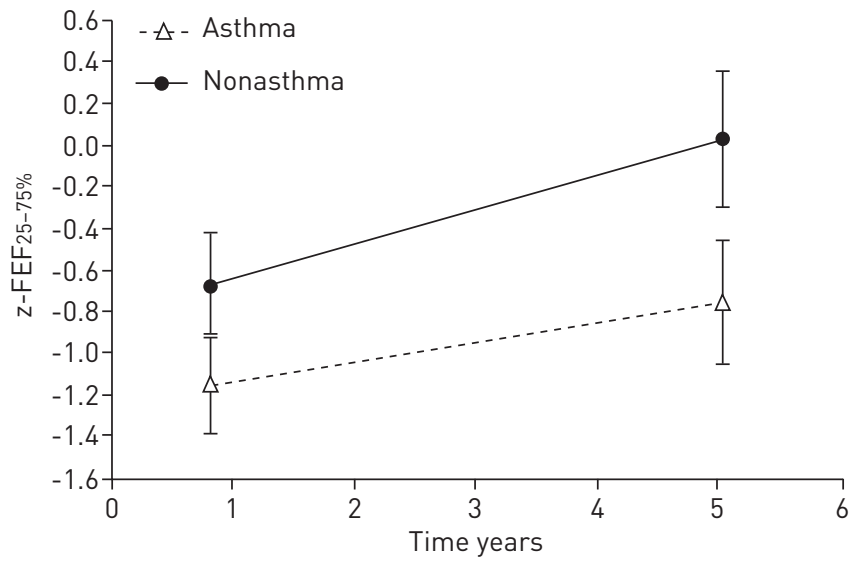

FIGURE 2 Z-score for forced expiratory flows at $25-75 \%$ of forced vital capacity (z-FEF25-75\%) at study entry and at 5 years of age. Subjects with asthma at 5 years of age had a statistically nonsignificant lower z-FEF25-75\% at entry to the study as infants prior to any wheezing ( $\mathrm{z}$-score difference: $0.46,95 \% \mathrm{CI}-0.01-0.93 ; \mathrm{p}=0.053$ ), and had significantly lower z-FEF $25-75 \%$ at 5 years of age compared with subjects without asthma (z-score difference: $0.78,95 \%$ CI $0.16-1.39$; $\mathrm{p}=0.014)$. In addition, the increase in $\mathrm{z}-\mathrm{FEF} 25-75 \%$ between infancy and 5 -year-old follow-up was smaller, but statistically nonsignificant, for subjects with asthma at 5 years of age compared with subjects without asthma (slope difference: 0.31 , 95\% CI $-0.01-0.60 ; \mathrm{p}=0.061$ ). Data are presented as meantSE. The model was adjusted by age at entry to the study, sex, race and smoking during pregnancy.

5-year-olds compared with subjects without asthma (z-score difference: 0.78, 95\% CI 0.16-1.39; $\mathrm{p}<0.014$ ). The increase in $\mathrm{z}-\mathrm{FEF} 25-75 \%$ between infancy and follow-up at 5 years of age was smaller, but statistically nonsignificant, between subjects with asthma as 5-year-olds compared with subjects without asthma (slope difference: $0.31,95 \%$ CI $-0.01-0.60 ; \mathrm{p}=0.061$ ) (fig. 2). Higher $\mathrm{z}-\mathrm{FEF} 25-75 \%$ at study entry was statistically nonsignificantly associated with a lower risk of asthma as a 5-year-old (OR 0.6, 95\% CI 0.36-1.001; $\mathrm{p}=0.051$ ). Lower $\mathrm{z}-\mathrm{FEF} 25-75 \%$ at study entry was significantly associated with lower $\mathrm{z}-\mathrm{FEF} 25-75 \%$ (OR 0.34 , $95 \%$ CI $0.06-0.61 ; \mathrm{p}=0.018)$ and greater airway reactivity $(\% \Delta \mathrm{FEV} 1)$ as a 5 -year-old $(\mathrm{OR} 5.15,95 \% \mathrm{CI}$ $0.39-9.92 ; \mathrm{p}<0.035)$.

\section{Airway reactivity}

Subjects with asthma as 5 -year-olds were no different from nonasthmatic subjects in terms of airway reactivity at entry to the study (PC30) (PC30 difference: 0.04, 95\%CI $-0.72-0.80 ; \mathrm{p}=0.904$ ); however, they had

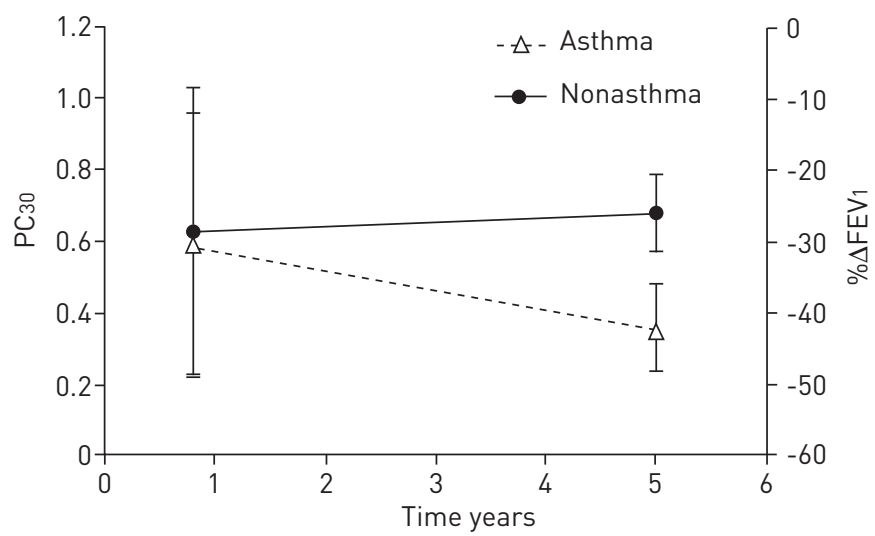

FIGURE 3 Airway responsiveness at study entry and at 5 years of age. Subjects with asthma at 5 years of age had no differences in airway reactivity at entry to the study (the provocative concentration decreasing baseline forced expiratory flow at $75 \%$ of forced vital capacity by $30 \%$ (PC30)) (PC30 difference: $0.04,95 \%$ CI $-0.72-0.80 ; p=0.904$ ), but greater airway reactivity at 5 years of age (percentage decrease in forced expiratory volume in $1 \mathrm{~s}\left(\mathrm{FEV}_{1}\right)$ from baseline $(\% \Delta \mathrm{FEV} 1)$ ) compared with subjects without asthma (\% $\Delta \mathrm{FEV} 1$ difference: $24.2 \%, 95 \%$ CI $7.6-40.8 \%$; $=0.005)$. In addition, the increase in airway reactivity between infancy and 5-year-old follow-up was significantly greater for subjects with asthma compared with subjects without asthma (slope difference: $16.1,95 \%$ CI 5.54-26.70; $\mathrm{p}=0.004$ ). Data are presented as mean \pm SE. The model was adjusted by age at entry to the study, sex, race and smoking during pregnancy. 
greater airway reactivity at 5 years of age compared with subjects without asthma (\% $\Delta \mathrm{FEV} 1$ difference: $24.2 \%$, 95\% CI 7.6-40.8\%; $\mathrm{p}=0.005$ ). The increase in airway reactivity between infancy and follow-up at 5 years of age was significantly greater for subjects with asthma compared with subjects without asthma (slope difference: 16.1, 95\% CI 5.54-26.70; $\mathrm{p}=0.004$ ) (fig. 3). Greater airway reactivity (lower PC30) at study entry was not associated with an increased risk for asthma at 5 years of age (OR 0.98, 95\% CI 0.66-1.44; $\mathrm{p}=0.899$ ), nor with greater airway reactivity $(\% \Delta \mathrm{FEV} 1)$ as a 5 -year-old (OR 4.34, 95\% CI -0.89-9.57; $\mathrm{p}=0.329)$.

\section{Infant immune characteristics and SCORAD}

Upon entry to the study, sensitisation to egg (OR 20.8, 95\% CI 1.61-250.00; $\mathrm{p}=0.020$ ) and higher SCORAD (OR 1.095, 95\% CI 1.01-1.19; $\mathrm{p}<0.026$ ) were associated with a significant risk for asthma at 5 years of age. There were no significant associations between any other specific allergen sensitisations, total IgE, or cytokine production by stimulated PBMCs with an increased risk for asthma at 5 years of age. Infant sensitisation to egg, higher total serum IgE, and higher IL-9 and IL-17 production were all significantly associated with higher eNO at 5 years of age (table 2). Sensitisation to egg and higher total IgE, as well as higher IL-17 and SCORAD, were all associated with lower FEF25-75\% at 5 years of age. There were no associations between immune characteristics in infants and airway reactivity at 5 years of age.

\section{Discussion}

In our selected cohort of infants with eczema, we found that increasing eNO prior to any episodes of wheezing, was associated with an increased risk of asthma and increasing airway reactivity at 5 years of age. Those with asthma at 5 years of age also had higher eNO at study entry, and a greater increase in eNO between infancy and follow-up compared with those without asthma. Egg sensitisation and increasing total IgE during infancy were also associated with an increased risk of asthma, higher eNO and lower FEF as a 5-year-old. Our findings suggest that, among infants at high risk of developing asthma, eNO levels early in life may provide important insights into the subsequent risk of asthma and its airway characteristics. In addition, our results emphasise that strategies to modulate the development of preschool asthma may require interventions very early in life.

Our study is the first to demonstrate that higher eNO measured prior to episodes of wheezing is not only associated with an increased risk of asthma as a preschool child, but is also associated with greater airway reactivity. Our current findings are consistent with our earlier report for this cohort that increasing eNO was associated with increasing airway reactivity when assessed at entry to study [14]. In an unselected birth cohort, LATZIN et al. [9] reported that higher eNO at 1 month of age was associated with an increased risk of severe respiratory symptoms in the first year of life among infants of atopic mothers or mothers who smoked tobacco during pregnancy. However, in another unselected cohort, GabRIELE et al.

TABLE 2 Immune characteristics and SCORAD at study entry as predictors of airway function at 5 years of age

\begin{tabular}{|c|c|c|c|c|c|c|}
\hline \multirow{2}{*}{ Parameter at study entry } & \multicolumn{6}{|c|}{ Parameter at 5 years of age } \\
\hline & Estimate $(95 \% \mathrm{CI})$ & p-value & Estimate $(95 \% \mathrm{CI})$ & p-value & Estimate $(95 \% \mathrm{CI})$ & p-value \\
\hline Egg allergen sensitisation & $16.7(6.48-26.94)$ & 0.002 & $-0.7(-1.44--0.08)$ & 0.029 & $-6.8(-20.28-6.63)$ & 0.313 \\
\hline Aeroallergen sensitisation ${ }^{\#}$ & $2.4(-8.51-13.26)$ & 0.664 & $0.5(-0.15-1.13)$ & 0.132 & $3.8(-8.02-15.67)$ & 0.518 \\
\hline Total Ig $\mathrm{E}^{\mathbb{T}}$ & $3.4(0.91-5.96)$ & 0.008 & $-0.2(-0.34--0.03)$ & 0.021 & $-1.3(-4.40-1.77)$ & 0.395 \\
\hline IL-9/IFN $\gamma^{+}$ & $6.2(0.12-12.30)$ & 0.046 & $-0.2(-0.61-0.12)$ & 0.181 & $0.4(-5.70-6.53)$ & 0.891 \\
\hline IL-10/IFN $\gamma^{+}$ & $8.7(-2.42-19.89)$ & 0.122 & $-0.3(-1.01-0.32)$ & 0.306 & $-7.3(-18.01-3.49)$ & 0.180 \\
\hline IL-13/IFN $\gamma^{+}$ & $4.5(-1.53-10.46)$ & 0.141 & $-0.2(-0.54-0.16)$ & 0.281 & $1.25(-4.50-7.00)$ & 0.663 \\
\hline IL-17/IFN $\gamma^{+}$ & $8.6(2.33-14.80)$ & 0.008 & $-0.4(-0.77--0.01)$ & 0.044 & $4.0(-2.98-10.98)$ & 0.253 \\
\hline SCORAD & $0.53(-0.10-1.15)$ & 0.097 & $-0.04(-0.08--0.002)$ & 0.037 & $-0.12(-0.97-0.74)$ & 0.780 \\
\hline
\end{tabular}

Bold font indicates statistical significance. SCORAD: Scoring Atopic Dermatitis; eNO: exhaled nitric oxide; z-FEF25-75\%: z-Score for forced expiratory flows at $25-75 \%$ of forced vital capacity; $\% \Delta F E V_{1}$ : percentage change in forced expiratory volume in $1 \mathrm{~s}$ between baseline and after final methacholine dose when reaching PC40,R5 (the methacholine dose that increased baseline R5 by 40\%); IL: interleukin; IFN: interferon. \#: presence of at least one positive sensitisation to the following allergens: Timothy grass, Bermuda grass, Short ragweed, Alternaria species and Mountain cedar; " : total serum IgE was expressed as the log transformed value; ${ }^{+}:$standardised as log(cytokine+1)/sD×(cytokine+1). The model was adjusted by age at entry to the study, sex, race and smoking during pregnancy. 
[10] reported that eNO measured at 6 months of age was only minimally associated with an increase in the risk of wheezing in the second year of life. Recently, Singer et al. [24] reported that among preschool children with recurrent respiratory symptoms, those with higher eNO (off-line) had an increased risk of asthma at school age. However, Debley et al. [25] reported that among infants with recurrent wheezing, elevated eNO was associated with lower FEF and greater bronchodilator responsiveness at 6-month follow-up. Evaluating a group of infants with recurrent wheezing, KotANIEMI-SYRJÄNEN et al. [26] found that increased eNO was associated with heightened airway reactivity. Cumulatively, these studies suggest that measuring eNO early in life may provide important insights into the subsequent risk of asthma and its airway characteristics.

In our current study, lower FEF during infancy were statistically nonsignificantly associated with a higher risk of developing asthma as a 5-year-old ( $\mathrm{p}=0.051)$, and those with asthma had a statistically nonsignificant smaller increase in FEF between infancy and follow-up compared with those without asthma $(p=0.061)$. Our results support the concept that the deficit in airway function associated with childhood asthma is present very early in life [5,27,28], and they are consistent with those of BISGAARD et al. [8] who followed up a selected high-risk cohort at 7 years of age. Our study suggests that these relationships are present even earlier, at the preschool age. As neither study evaluated post-bronchodilator spirometry during infancy and at follow-up, it remains unclear whether the lower flows early in life, as well as the worsening with time, are related to fixed, non-reversible airway dysfunction or increased airway tone. At 4-year follow-up of our cohort, the lower spirometry in subjects with asthma was no longer present following a bronchodilator [4]. We also found that lower spirometry during infancy was associated with a greater bronchodilator response at 4 years of age, which suggested an increase in airway tone, rather than fixed airway obstruction or abnormal lung growth. It remains important to determine whether decrements in airway function early in life and greater decrements in childhood represent changes in lung growth, worsening fixed airway disease or increased airway tone, as these distinctions are critical in determining the potential importance of early intervention and evaluating outcomes.

In our study, we did not find association between airway reactivity during infancy and risk of asthma at 5 years of age. Our results differ from those of BisGAARD et al. [8], who found that increased sensitivity to $\mathrm{MCh}$ early in life was associated with an increased risk of asthma at 7 years of age. This difference may result from differences in the selection of high-risk subjects, the smaller number of subjects in our study and the differing methodologies used to assess airway reactivity. The only other longitudinal study of airway reactivity from early in life evaluated an unselected cohort; however, the relationship of airway reactivity in infancy to asthma or respiratory symptoms at follow-up was not consistent among follow-up studies $[28,29]$.

Egg sensitisation as an infant was the most important risk factor for asthma, higher eNO, lower spirometry and atopy at 5 years of age, while total serum IgE and SCORAD were less consistent and less important than egg sensitisation. These findings are consistent with our previous report that food sensitisation during infancy was associated with asthma and lung function at 4 years of age [4], and consistent with other cohort studies demonstrating the importance of food allergies early in life, while aeroallergen sensitisation does not become important until several years of age [30-33].

We did not find that cytokine production by stimulated PBMCs was associated with asthma at 5 years of age, which contrasts with our previous findings that increasing IL-4 and IL-10 as an infant was associated with an increased risk of asthma at 4 years of age [4]. However, we did find that increasing IL-17 production at entry to the study was associated with lower lung function and higher eNO at 5 years of age. This finding is consistent with studies finding increased IL-17 in asthmatic airways, and the ability of IL-17 to promote airway hyperresponsiveness [34-37]. In addition, in children with asthma, serum IL-17 levels were positively correlated with eNO [38]. IL-17 has been proposed to be an effector cytokine that mediates steroid resistant pulmonary inflammation [39]. The function of IL-17 in infants has not been examined and our study suggests it might have unique functions early in life.

There are several limitations to our study. Our subjects had eczema, which may explain our high prevalence of asthma (68\%) at 5 years of age; therefore, our results cannot be extrapolated to a general population. In addition, our subjects were not a birth cohort; subjects who were older at entry to the study had gone longer without any wheezing illness. We adjusted our analysis for age at entry, which did not have a significant effect; therefore, we do not believe this created a sampling bias that affected the interpretation of our results. We found that severity of eczema at study entry was associated with an increased risk of asthma at 5 years of age, which is consistent with previous studies [20, 40]; however, we may have underestimated this association. For infants, SCORAD was assessed at the time of lung function testing and not at the time of eczema diagnosis; therefore, subjects were already receiving treatment for their dermatitis. We found that 22 subjects did not perform acceptable spirometry at 5 years of age; 
however, this is consistent with assessment at this young age [17]. For the same reason, we assessed airway reactivity using IOS, as well as using the change in spirometry from baseline to the last $\mathrm{MCh}$ dose. Including spirometry was important as an outcome, as it proved to be more sensitive than oscillometry in discriminating baseline airway function and airway reactivity in those with and without asthma at 5 years of age, which is consistent with several studies of children $[20,40]$.

In summary, we found that eNO obtained prior to any episodes of wheezing was associated with an increased risk of asthma and/or altered airway function as a preschool child. Those with asthma at 5 years of age had a greater increase in eNO and airway reactivity between infancy and follow-up compared with those without asthma. These findings indicate that the airway characteristics of asthma are present very early in life and progress by 5 years of age. eNO and spirometric measurements during infancy may be useful in evaluating strategies to modify the progression of asthma early in life.

\section{References}

1 Pijnenburg MWH, De Jongste JC. Exhaled nitric oxide in childhood asthma: a review. Clin Exp Allergy 2008; 38: 246-259.

2 Weiss ST, Van Natta ML, Zeiger RS. Relationship between increased airway responsiveness and asthma severity in the childhood asthma management program. Am J Respir Crit Care Med 2000; 162: 50-56.

3 Tantisira KG, Fuhlbrigge AL, Tonascia J, et al. Bronchodilation and bronchoconstriction: predictors of future lung function in childhood asthma. J Allergy Clin Immunol 2006; 117: 1264-1271.

4 Sarria EE, Mattiello R, Yao W, et al. Atopy, cytokine production, and airway reactivity as predictors of pre-school asthma and airway responsiveness. Pediatr Pulmonol 2014; 49: 132-139.

5 Martinez FD, Wright AL, Taussig LM, et al. Asthma and wheezing in the first six years of life. The Group Health Medical Associates. N Engl J Med 1995; 332: 133-138.

6 Young S, Le Souëf PN, Geelhoed GC, et al. The influence of a family history of asthma and parental smoking on airway responsiveness in early infancy. $N$ Engl J Med 1991; 324: 1168-1173.

7 Stern DA, Morgan WJ, Wright AL, et al. Poor airway function in early infancy and lung function by age 22 years: a non-selective longitudinal cohort study. Lancet 2007; 370: 758-764.

8 Bisgaard H, Jensen SM, Bønnelykke K. Interaction between asthma and lung function growth in early life. Am J Respir Crit Care Med 2012; 185: 1183-1189.

9 Latzin P, Kuehni CE, Baldwin DN, et al. Elevated exhaled nitric oxide in newborns of atopic mothers precedes respiratory symptoms. Am J Respir Crit Care Med 2006; 174: 1292-1298.

10 Gabriele C, Jaddoe VW, van Mastrigt E, et al. Exhaled nitric oxide and the risk of wheezing in infancy: the Generation R Study. Eur Respir J 2012; 39: 567-572.

11 Martínez T, Weist A, Williams T, et al. Assessment of exhaled nitric oxide kinetics in healthy infants. J Appl Physiol 2003; 94: 2384-2390.

12 American Thoracic Society, European Respiratory Society. ATS/ERS recommendations for standardized procedures for the online and offline measurement of exhaled lower respiratory nitric oxide and nasal nitric oxide, 2005. Am J Respir Crit Care Med 2005; 171: 912-930.

13 Silkoff PE, Erzurum SC, Lundberg JO, et al. ATS workshop proceedings: exhaled nitric oxide and nitric oxide oxidative metabolism in exhaled breath condensate. Proc Am Thorac Soc 2006; 3: 131-145.

14 Tepper RS, Llapur CJ, Jones MH, et al. Expired nitric oxide and airway reactivity in infants at risk for asthma. J Allergy Clin Immunol 2008; 122: 760-765.

15 Jones M, Castile R, Davis S, et al. Forced expiratory flows and volumes in infants. Normative data and lung growth. Am J Respir Crit Care Med 2000; 161: 353-359.

16 Eigen H, Bieler H, Grant D, et al. Spirometric pulmonary function in healthy preschool children. Am J Respir Crit Care Med 2001; 163: 619-623.

17 Beydon N, Davis SD, Lombardi E, et al. An official American Thoracic Society/European Respiratory Society statement: pulmonary function testing in preschool children. Am J Respir Crit Care Med 2007; 175: 1304-1345.

18 Crapo RO, Casaburi R, Coates AL, et al. Guidelines for methacholine and exercise challenge testing - 1999. This official statement of the American Thoracic Society was adopted by the ATS Board of Directors, July 1999. Am J Respir Crit Care Med 2000; 161: 309-329.

19 Vink GR, Arets H, van der Laag J, et al. Impulse oscillometry: a measure for airway obstruction. Pediatr Pulmonol 2003; 35: 214-219.

20 Schulze J, Smith HJ, Fuchs J, et al. Methacholine challenge in young children as evaluated by spirometry and impulse oscillometry. Respir Med 2012; 106: 627-634.

21 Yao W, Barbé-Tuana FM, Conrado JL, et al. Evaluation of airway reactivity and immune characteristics as risk factors for wheezing early in life. J Allergy Clin Immunol 2010; 126: 483-488.

22 Jenmalm MC, Aniansson-Zdolsek H, Holt PG, et al. Expression of and responses to CD2 and CD3 in 18-month-old children with and without atopic dermatitis. Pediatr Allergy Immunol 2000; 11: 175-182.

23 Pucci N, Novembre E, Cammarata MG, et al. Scoring atopic dermatitis in infants and young children: distinctive features of the SCORAD index. Allergy 2005; 60: 113-116.

24 Singer F, Luchsinger I, Inci D, et al. Exhaled nitric oxide in symptomatic children at preschool age predicts later asthma. Allergy 2013; 68: 531-538.

25 Debley JS, Stamey DC, Cochrane ES, et al. Exhaled nitric oxide, lung function, and exacerbations in wheezy infants and toddlers. J Allergy Clin Immunol 2010; 125: 1228-1234.

26 Kotaniemi-Syrjänen A, Malmberg LP, Malmström K, et al. Factors associated with elevated exhaled nitric oxide fraction in infants with recurrent respiratory symptoms. Eur Respir J 2013; 41: 189-194.

27 Young S, Arnott J, Le Souef PN, et al. Flow limitation during tidal expiration in symptom-free infants and the subsequent development of asthma. J Pediatr 1994; 124: 681-688.

28 Turner SW, Palmer LJ, Rye PJ, et al. The relationship between infant airway function, childhood airway responsiveness, and asthma. Am J Respir Crit Care Med 2004; 169: 921-927. 
Palmer LJ, Rye PJ, Gibson NA, et al. Airway responsiveness in early infancy predicts asthma, lung function, and respiratory symptoms by school age. Am J Respir Crit Care Med 2001; 163: 37-42.

30 Rhodes HL, Sporik R, Thomas P, et al. Early life risk factors for adult asthma: a birth cohort study of subjects at risk. J Allergy Clin Immunol 2001; 108: 720-725.

31 Kjaer HF, Eller E, Andersen KE, et al. The association between early sensitization patterns and subsequent allergic disease. The DARC birth cohort study. Pediatr Allergy Immunol 2009; 20: 726-734.

32 Kulig M, Bergmann R, Klettke U, et al. Natural course of sensitization to food and inhalant allergens during the first 6 years of life. J Allergy Clin Immunol 1999; 103: 1173-1179.

33 Illi S, von Mutius E, Lau S, et al. The pattern of atopic sensitization is associated with the development of asthma in childhood. J Allergy Clin Immunol 2001; 108: 709-714.

34 Al-Ramli W, Préfontaine D, Chouiali F, et al. T(H)17-associated cytokines (IL-17A and IL-17F) in severe asthma. J Allergy Clin Immunol 2009; 123: 1185-1187.

35 Lajoie S, Lewkowich IP, Suzuki Y, et al. Complement-mediated regulation of the IL-17A axis is a central genetic determinant of the severity of experimental allergic asthma. Nat Immunol 2010; 11: 928-935.

36 Kudo M, Melton AC, Chen C, et al. IL-17A produced by $\alpha \beta \mathrm{T}$ cells drives airway hyper-responsiveness in mice and enhances mouse and human airway smooth muscle contraction. Nat Med 2012; 18: 547-554.

37 Pham D, Sehra S, Sun X, et al. The transcription factor Etv5 controls T17 cell development and allergic airway inflammation. J Allergy Clin Immunol 2014; 134: 204-214.

38 Chien JW, Lin CY, Yang KD, et al. Increased IL-17A secreting CD4 ${ }^{+} \mathrm{T}$ cells, serum IL-17 levels and exhaled nitric oxide are correlated with childhood asthma severity. Clin Exp Allergy 2013; 43: 1018-1026.

39 McKinley L, Alcorn JF, Peterson A, et al. TH17 cells mediate steroid-resistant airway inflammation and airway hyperresponsiveness in mice. J Immunol 2008; 181: 4089-4097.

40 Delacourt C, Lorino H, Herve-Guillot M, et al. Use of the forced oscillation technique to assess airway obstruction and reversibility in children. Am J Respir Crit Care Med 2000; 161: 730-736. 